\title{
Theory of existence and uniqueness for the nonlinear Maxwell-Boltzmann equation
}

\section{Aleksander Glikson}

A review of the development of the theory of existence and uniqueness of solutions to initial-value problems for mostly reduced versions of the nonlinear Maxwell-Boltzmann equation with a cut-off of intermolecular interaction, precedes the formulation and discussion of a somewhat generalized initial-value problem for the full nonlinear Maxwell-Boltzmann equation, with or without a cut-off. This is followed by a derivation of a new existence-uniqueness result for a particular Cauchy problem for the full nonlinear Maxwell-Boltzmann equation with a cut-off, under the assumption that the monatomic Boltzmann gas in the unbounded physical space $X$ is acted upon by a member of a broad class of external conservative forces with sufficiently wellbehaved potentials, defined on $X$ and bounded from below. The result represents a significant improvement of an earlier theorem by this author which was until now the strongest obtained for Cauchy problems for the full Maxwell-Boltzmann equation. The

Received 14 January 1977. The author is grateful to Dr R.G. Muncaster and Professor C.A. Truesdell for allowing him the opportunity of reading and commenting on some drafts of those parts of their new book [28] which contain, or are related to, some of his results and ideas as presented in [9], [10], and the present paper. He is also grateful to them for making available a draft of other selected chapters and sections of the book which are devoted to the theory of the Maxwell-Boltzmann equation. Thanks are also due to Professor Yasushi Shizuta and Professor J.-P. Guiraud for bringing some of the references to the author's attention, and to his colleagues at the University of New England for some improvements of the original wording. 
improvement is basically due to the introduction of equivalent norms in a Banach space, the definition of which is connected with an exponential function of the total energy of a freestreaming molecule.

\section{Introduction}

In this paper we shall mainly be concerned with the formulation, discussion, and solution of the general space-dependent initial-value problem for the full nonlinear Maxwell-Boltzmann equation ("MaxwellBoltzmann" in Hilbert's terminology). The Maxwell-Boltzmann equation is a specific attempt to describe the behaviour of a sufficiently rarefied ideal gas of identical monatomic molecules in terms of the evolution of the oneparticle distribution function $f$ having arguments $(t, x, \xi) \in T_{\infty} \times X \times \Xi, f(t, x, \xi) \geq 0$. Here $t$ is the time coordinate, $x, \xi$ are respectively the position vector and the velocity vector (of the so-called representative or test molecule) at instant $t, T_{\infty}$ is the time interval equivalent nondimensionally to $[0, \infty), X$ and $\Xi$ are respectively the physical (or position) space and the velocity space, and the set $X \times \equiv$ (equivalent to the phase space of the molecule) is nondimensionally $R^{3} \times R^{3}$.

The theory of existence and uniqueness of the solutions to the Cauchy problem for the Maxwell-Boltzmann equation is reasonably complete and well developed in the spatially homogeneous case (that is when $f$ is by assumption independent of $x$ ) for the initial condition $f(0, \xi)=f_{0}(\xi) \geq 0$ such that $f_{0} \in L^{I}(\Xi)$, assuming the absence of any field of external forces (denoted in the following by $F$ ). Global existence-uniqueness results (generalized or classical, in the meaning defined in Subsection 2.B of this paper) were obtained in this case for various classes of cut-off intermolecular interaction, while imposing additional restrictions on the initial datum $f_{0}$ (some of them quite natural from the physical point of view; for example, the requirement of the existence of further lower moments of $\left.f_{0}\right)$. See [11], [14], [1], [2], [16] for relevant literature, covering the results up to 1973, and for a brief description and discussion of these results. The published results 
were recently supplemented and improved by an interesting and important existence-uniqueness-boundedness-smoothness theorem (Truesdell and Muncaster [28]), being a continuation of, and complement to, Arkeryd's investigations [1] and [2]. The authors show that if $\left(1+\xi^{2}\right)^{2} f_{0} \in L^{1}(\equiv)$ and a cut-off is assumed, then there exists a unique function $f$, nonnegative and Lebesgue measurable on $T_{\infty} \times \Xi$, differentiable almost everywhere, satisfying the classical Cauchy problem for the reduced spatially homogeneous Maxwell-Boltzmann equation, and for which, for each $t^{*}=$ const $>0$, there exists $c_{t^{*}}=$ const $>0$ such that

$\left\|\left(1+\xi^{2}\right)^{2} f(t, \cdot)\right\|_{L^{I}(\Xi)} \leq c_{t^{*}}$ if $t \in\left[0, t^{*}\right]$.

Though the spatially homogeneous case is interesting for mathematical and general theoretical considerations, it does not correspond to realistic rarefied gas flows since the fluid dynamics for this case is trivial - the density, mean velocity, pressure tensor, heat flux, and so on, are spatially constant in the region occupied by the gas. For a long time, progress in the much more realistic (and much more complex) space-dependent Cauchy problem could not match that in the spatially homogeneous case, despite the fact that authors had always assumed a cut-off and the absence of external forces. The existence and uniqueness of solutions were shown for finite intervals of time of lengths (roughly speaking) inversely proportional to the degree of departure of the initial data from an absolute Maxwellian distribution. The most important existence-uniqueness theorems of that period proven for the Cauchy problem for some classes of initial conditions, and under the assumption of the so-called angularly cut-off hard potentials ([13], especially Sections II and III), were due to Grad [15]. See also his earlier papers [13], [14] for the important fundamentals of the mathematical theory of the reduced ( $F \equiv 0$ ) MaxwellBoltzmann equation, and [12] (especially Section IX) for the connection with fluid dynamics.

These investigations by Grad, based to a certain extent on the pioneering works of Hilbert, Carleman, Wild, and Morgenstern (for instance, Grad's monotone iteration procedure is based on that of Wild [31]), were in turn extensively used for further investigations of a variety of theoretical (mathematical) problems of the kinetic theory of gases 
connected with, or related to, the Maxwell-Boltzmann equation, such as in the work of Giraud and others in France (see for example [16], [17] and the references contained therein) and more recently in the work of Shizuta, Uka $i$, and others essentially done in Japan (see [27] and the references contained therein). The work done by these Japanese authors is of particular importance to us since it considers directly the initial-value problems for the space-dependent case under discussion. Shizuta and others, using many elements of Grad's approach (for example, the type of cut-off and the iterative scheme) as well as some elements of the theory of semigroups, extended Grad's local in time existence-uniqueness results (including the continuous dependence on the initial data) to global existence theorems for classical and/or generalized solutions, under the principal assumption of the closeness of the initial state of the gas to equilibrium. Since a more detailed description of their results would be quite lengthy, in view of the sophistication of both the mathematical methods and the notation used, and since some of the papers listed in [27] were not available to this author, the reader is referred to the originals for details. (Although an application of the methods of the theory of semigroups to Cauchy problems related to, or connected with, the MaxwellBoltzmann equation was initiated much earlier by Arsen'ev in [5], and a few years later developed significantly by Scharf in [24] and [25] - these three papers were basically on the linearized version of the equation.)

The above results for the space-dependent Cauchy problem are very similar to, or identical with, the ones obtained for a standard initialboundary value problem (briefly, "mixed problem") in a bounded domain $X_{B}(\subset X)$ which is a rectangular box with specularly reflecting walls. This boundary condition is, roughly speaking, almost equivalent to a periodic boundary condition in a smaller rectangular domain in $X$. (Both these conditions were first introduced into the existence theory somewhat unclearly by Morgenstern in [22, \$5].) For a precise description of the connection between the two types of boundary conditions, see [14, pp. 161-162].

Chronologically, the existence-uniqueness results for the mixed problem (derived by similar if not identical means by the same set of authors as listed above for the space-dependent Cauchy problem) always preceded corresponding results for "the whole of $X$ ". For example, for 
small initial deviations from an absolute Maxwellian distribution, significant results were obtained for the mixed problem by Ukai [29] and somewhat later by Shizuta [26] (strictly speaking, $X_{B}$ of [26] is cubic with a periodic boundary condition). Ukai extended Grad's local existence theorem for $X_{B}$ in a Sobolev space [14] to a global one in a larger Sobolev space with the same norm, while Shizuta was able to show that his global continuous solutions were smoother than Ukai's under less restrictive conditions on the initial data. Shizuta's approach relies heavily upon the theory of perturbed semigroups as well as on some elements of the previous investigations of Grad and the classical work of Carleman. (One cannot rule out the possibility that the significant success with this standard mixed problem may be linked in an intrinsic way with the wellknown result for the Boltzmann-Gibbs conjecture due to Sinai (for example see $[4, \S 18]$ and the references contained therein) that a gas of identical 'billiard-ball' molecules, confined to a container $X_{B}$ as specified above, is ergodic. Here "identical" means "satisfying (for $t \in T_{\infty}$ ) the same vector equation of motion under Axioms 1 and 2 of Subsection 2.A below".)

Before proceeding, it should be mentioned that in this review we omit all space-dependent existence-uniqueness results obtained for various modified forms of the reduced or full Maxwell-Boltzmann equation; for example, we omit a theorem due to Arkeryd [3]. We also omit some slight perturbations on the theory of the linearized Maxwell-Boltzmann equation, and we do not discuss results obtained for "the approach to equilibrium", that is more precisely, for the problem of uniform asymptotic stability in the large (for example, in the sense of Liapunov) of an appropriate absolute maxwellian distribution function. (The latest results obtained for this problem in the space-dependent case seem to be those of [26] and [27].)

In space-dependent Cauchy problems, involving a reasonably broad class of external forces acting on a general class of cut-off molecules in $X$, the only investigations made so far are those of [9] and [10]. In these respects the results of [9] and [10] for continuous solutions (generalized, regarding their smoothness) were until now the strongest obtained in the theory of existence and uniqueness of solutions to the full nonlinear Maxwell-Boltzmann equation. 
In Section 3 of this paper, we significantly strengthen the results of [9] and [10] by weakening certain restrictions imposed previously on finite intervals of existence and uniqueness of the solutions. (Only an improvement on [10] will be presented, since a similar improvement on [9] is fundamentally less useful.) The advance is basically due to the introduction of a family of equivalent norms in a certain Banach space, the definition of which is connected with an exponential function of the total energy of the free-streaming test molecule. This leads to the replacenent of some Lipschitz conditions, obtained in [9] and [10] for certain integral operators on closed subsets of the Banach space, by strict contractions on the same subsets. (As Grad pointed out in [11, p. 243] and [14, p. 155], one cannot be absolutely sure of ultimately obtaining general solutions of the nonlinear Maxwell-Boltzmann equation valid for all $t \in T_{\infty}$, but the nonlinear theory of the Navier-Stokes equations (which should arise as a special limiting case from the Maxwell-Boltzmann equation) helps one to believe in the positive outcome of the expectation.)

In Section 2, which is not only a preparation for the considerations of Section 3, but more importantly a preparation for further investigations, we discuss the general space-dependent Cauchy problem for the full MaxwellBoltzmann equation, with or without a cut-off. First, in Subsection 2.A, certain mathematical characteristics of the fundamental concept of the monatomic molecule, characteristics which in this author's opinion are definitely needed in the existence-uniqueness theory, are specified by laying down some mathematical axioms and definitions, while supplementing them by remarks and comments. (The word "fundamental" is used here in the meaning that the concept itself is not defined by the axioms.) In such a way we want to avoid the habitual lack of rigour and completeness of those theoretical preliminaries of most texts on kinetic theory (and more generally statistical mechanics), which by their very nature are necessary preliminaries to the construction of the theory of existence and uniqueness for the (full) Maxwell-Boltzmann equation.

It should be mentioned here that Subsection 2.A, being basically an extension and reworking of this author's general ideas and preliminaries from [9] and [10], exhibits some unavoidable similarities to certain portions of the text of [28] which are influenced to a varying extent by [9] and [10]. It is hoped that the reader will find the content and 
presentation of Subsection 2.A a useful complement to the related material of [28].

Other axioms, concepts, and laws which (again in this author's opinion) may not be necessarily needed in the theory of existenceuniqueness, and/or which are in general correctly given in many well-known texts and papers, are either omitted (for example, some of those used in the derivations of the Maxwell-Boltzmann equation; see [11]), given and briefly discussed in Subsection 2.B, or to be found in the literature to which references are made in the text.

\section{The general space-dependent initial-value problem}

\section{A. PRELIMINARIES}

AXIOM 1. There is a vector-differential equation, the newtonian (vector) equation of motion,

$$
\frac{d}{d t}(x, \xi)=(\xi, F(t, x, \xi)),
$$

obeyed by each monatomic molecule (of a given rarefied gas). Here $x=x(t)$ and $\xi=\xi(t)$ are, for any fixed $t \in T \equiv\left[t_{0}, t_{1}\right]$, points of $X$ and $\Xi$ respectively, given in cartesian coordinates $(X$ and $\Xi$ are as in Section $1 ; t_{0}$ and $t_{1}$ are constants, $\left.t_{1}>t_{0}\right)$; the function $F$ is supposed to be given, defined everywhere on $R_{T}^{7} \equiv T \times X \times \Xi$, and sufficiently well-behaved on $R_{T}^{\top}$ (see Axiom 2); $\mathrm{F}(t, \mathrm{x}, \xi)$, often denoted for brevity as $F$, is the so-called (resultant) external force per unit mass acting on the molecule 'positioned' at $(t, x, \xi) \in R_{T}^{7}$.

REMARK. A purist should also postulate the existence of mass of the molecule as a positive constant assigned to the molecule; that is, mass is an absolute invariant over $T_{\infty} \times X \times \Xi$. In (2.1) mass has been implicitly taken account of in $F$. (Now, corresponding to $t_{0}, T_{\infty} \equiv\left[t_{0}, \infty\right)$.)

AXIOM 2. For the function $F$, there exists $S: T \times R_{T}^{7}+X \times \Xi$ and such that, for each fixed $\left(s, x_{s}, \xi_{8}\right) \in R_{T}^{T}$ and all $t \in T$, 
$(x, \xi)=S\left(t, s, x_{s}, \xi_{s}\right)$ is a unique solution of (2.1) satisfying the initial condition $S\left(s, 8, x_{8}, \xi_{8}\right)=\left(x_{8}, \xi_{8}\right)$.

REMARK. Physically, such a solution is postulated to describe for each $t \in T$ (by one-to-one correspondence) the so-called classical state of the molecule in the molecule's phase space.

REMARKS 2.1. Sets of functions $F$ allowed by Axioms 1 and 2 follow from various classical existence and/or uniqueness theorems (in particular those associated with the names of Cauchy, Peano, Picard, Lindelöf, Kamke, Osgood, Nagumo, and Perron) which may be found in most standard texts on differential equations; for newer results, see for example [20], $\S 1.3$, $1.6,1.8$, and the references contained therein. Usually these theorems are proved for local existence and uniqueness. When generalizing such local theorems to the case specified by the above axioms, in which the domain $R_{T}^{7}$ is unbounded and with a general $T$, there can be of course immediate trivial extensions, as for example the one given in [18] (p. 53, footnote 1) for the Picard-Lindelöf theorem. More importantly though, with some effort the existence and uniqueness can be often shown valid for the unbounded domain under weaker conditions than those in trivial extensions, and such extensions are more useful in our case. (For example, in the case of the Picard-Lindelof theorem it would be the uniform Lipschitz continuity of $F$ in $(x, \xi)$ in the whole of $R_{T}^{7}$.)

LEMMA 2.1. $S$ is a continuous function on $T \times R_{T}$. DEFINITIONS 2.1. For a fixed $\left(s, x_{s}, \xi_{s}\right) \in R_{T}^{7}$, the set $\Gamma_{T}\left(s, x_{s}, \xi_{s}\right)=\left\{\right.$ all points $\left.(t, x, \xi) \mid(x, \xi)=S\left(t, s, x_{s}, \xi_{s}\right) ; t \in T\right\}$ is called the free-streconing $R_{T}^{T}$-trajectory (of the molecule) through $\left(s, x_{s}, E_{s}\right)$. If $T$ can be replaced by $T_{\infty} \equiv\left[t_{0}, \infty\right)$, we have the whole 'positive' trajectory denoted by $\Gamma_{\infty}\left(s, x_{s}, \xi_{s}\right)$.

REMARK. In the theory of differential equations or in statistical mechanics a trajectory would usually be defined on $X \times \Xi$ or an equivalent 
space. However, for our purposes Definitions 2.1 are more useful since

(i) the distribution function $f$ and related functions of interest are in general defined on $R_{T}^{7}$, and

(ii) the definitions help to avoid some ambiguities (for example, see Definitions 2.2 and 2.3 below, or general conments at the end of this subsection).

DEFINITIONS 2.2. We say that a real scalar-valued function $g$ of variables $t, x, \xi$ is differentiable in some sense (for example, almost everywhere) along a trajectory $\Gamma_{T}\left(s, x_{s}, \xi_{s}\right)$, if

$$
D g(t, x, \xi) \equiv \frac{d g(t, x(t) \xi(t))}{d t}
$$

exists in the same sense for $(x, \xi)=S\left(t, s, x_{s}, \xi_{g}\right)$ and $t_{0} \leq t \leq t_{1}$. If the function $g$ is differentiable along each $\Gamma_{T}\left(s, x_{s}, \xi_{g}\right)$, that is for an arbitrary $\left(s, x_{s}, \xi_{g}\right) \in R_{T}^{7}$, then we say that $g$ is differentiable along the free-streaming trajectories in $R_{T}^{7}$ or, briefly, differentiable along the $R_{T}^{7}$-trajectories. We call $\operatorname{Dg}(t, \mathrm{x}, \xi)$ the total derivative of $g(t, x, \xi)$ with respect to $t$ (briefly, the total (time) derivative of $g)$, and usually denote it by $D g$. Naturally, $\left.D g\right|_{t=t_{0}}$ and $\left.D g\right|_{t=t_{1}}$ are taken as the right- and left-hand side derivatives, respectively.

REMARK 2.2. If $g$ of Definitions 2.2 is differentiable on $R_{T}^{7}$, $(2.2)_{1,2} \quad D g=((\partial / \partial t)+\xi .(\partial / \partial x)+F \cdot(\partial / \partial \xi)) g \equiv D_{d} g$.

REMARKS 2.3. The notation $D$ of [9] and [10], as well as the related terminology of those papers (like "Dg exists" or " $g$ has the total derivative") were used there with the same meaning as that given in Definitions 2.2, but, since we followed the use of the rather ambiguous notation of Grad [11], [13], [14], and of many others (for example, see [19], Equation 2.9 and subsequent statements, or see [7], §3.1), we automatically 'specified' $D g$ in the form of $D \mathscr{g}$, although like Grad himself we did not mean to imply by such a notation that any of the 
separate partial derivatives $\partial g / \partial t, \partial g / \partial x$, or $\partial g / \partial \xi$ need exist independently. In this paper we use notation clearly distinguishing functions $g$ for which $D g$ is defined (in some sense) from those for which the separate derivatives exist and so $D_{d^{g}}$ is defined; see also Remark 2.4. (A tendency to treat the operators $D$ and $D_{d}$ as equivalent is not confined to kinetic theory texts. For example, such equivalence is taken for granted in almost all texts of fluid mechanics.) We should also stress that except in [28] the importance of Axiom 2 in writing (2.2) 1 has not been pointed out in previous literature on kinetic theory.

REMARK 2.4. It is worth recalling that the mere existence of all partial derivatives of a continuous function of several variables, plus the uniqueness and differentiability of parametric representations of these variables, is not in general sufficient for the equivalence of the total derivative at the parameter value, $t=t^{*}$ say, and the 'chain-rule' derivative formally evaluated at $t=t^{*}$. A classical illustration is given by $g: R^{2} \rightarrow R$ such that $g(x, y)=x y^{2} /\left(x^{2}+y^{2}\right)$ for $x^{2}+y^{2} \neq 0$, $g(0,0)=0$, with $x=y=t$ and $t^{*}=0$.

DEFINITIONS 2.3. The operator $\{\cdot\}_{t}^{s}$, if acting on functions with domain $R_{T}^{7}$, is defined by

$$
\left\{g\left(s, x_{s}, \xi_{s}\right)\right\}_{t}^{s}=g(t, x, \xi),
$$

where $s, t \in T$ and $(x, \xi)=s\left(t, s, x_{s}, \xi_{s}\right)$. It will be called the trajectorial shifter along $\Gamma_{T}\left(s, x_{s}, \xi_{s}\right)$. The 'inverse' operator, $[\cdot\}_{s}^{t}$, which can be shown to exist on the basis of the above axioms, is defined by

$$
\{g(t, x, \xi)\}_{s}^{t}=g\left(s, x_{s}, \xi_{s}\right),
$$

where $\left(x_{s}, \xi_{s}\right)=s(s, t, x, \xi)$; that is $\left(x_{s}, \xi_{s}\right)$ is a unique solution to the vector equation

$$
\frac{d}{d s}\left(x_{s}, \xi_{s}\right)=\left(\xi_{s}, F\left(s, x_{s}, \xi_{s}\right)\right)
$$


with initial conditions $x_{s}(s=t)=x, \quad \xi_{s}(s=t)=\xi$. (of course, $\{\cdot\}_{S}^{t}$ may be interpreted as the trajectorial shifter along $\left.\Gamma_{T}(t, x, \xi).\right)$ We shall often write $\{g\}_{s}^{t}$ instead of $\{g(t, x, \xi)\}_{s}^{t}$, if it does not lead to any confusion.

REMARK. The distinction between the trajectorial shifter and the operator, $S_{t, s}$ say, of translating along the $(X \times \Xi)$-trajectories [20] is clearly seen from the definition of $s_{t, s}$, namely, $s_{t, s}\left(x_{s}, \xi_{s}\right)=(x, \xi)$.

REMARKS 2.5. $\{g\}_{t}^{t} \equiv g$ and, for any $g$ defined everywhere on $R_{T}^{7}$, $\left\{\{g\}_{s}^{t}\right\}_{t}^{s} \equiv g$. The assumption that $\operatorname{dom} g \equiv R_{T}^{7}$ is important; for example, see $\left\{\{x \cdot \xi /(t-1)\}_{1}^{t}\right\}_{t}^{1}$ for $F \equiv 0$.

REMARK 2.6. If $D g$ exists for $t \in T$, then

$$
\begin{gathered}
D_{g}=\lim _{\zeta \rightarrow 0} \zeta^{-1}\left(\{g\}_{t+\zeta}^{t}-\{g\}_{t}^{t}\right)=\left(\partial\{g\}_{t+\zeta}^{t} / \partial \zeta\right)_{\zeta=0} ; \\
\zeta=0^{+}, 0^{-} \text {for } t=t_{0}, t_{1} \text { respectively. }
\end{gathered}
$$

This explains the connection between $D$ and $\{\cdot\}_{u}^{t}$, and gives $D g$ as a function on $R_{T}^{7}$.

LEMMA 2.2. It follows from Lemma 2.1 that for $s, t \in T$ a trajectorial shifter maps continuous functions, defined on $R_{T}^{T}$, into continuous functions.

LEMMA 2.3. If $s$ is independent of $t$, then $D\{g\}_{s}^{t} \equiv 0$ wherever $D\{g\}_{s}^{t}$ exists.

REMARK. It seems proper to explain here that the condition $t \neq s$ in the property (12b) of [9] was meant to express the above assumption of independence between $s$ and $t$ by pointing out that $s$ and $t$ are two 
different (in the sense of independent) variables. The assumption is needed, see the case $\{g\}_{t}^{t}$ of Remarks 2.5 or see Remark 2.6.

GENERAL COMMENTS. It is clear from the preceding that the explicit presentation of Axiom 2 is needed to assure rigour and completeness in various parts of kinetic theory, and more generally of statistical mechanics. As a rule, this has not been recognized in the literature, though one may sometimes trace this very axiom used implicitly in a statement like "no trajectories can intersect one another since then for the same initial condition we would have two different histories of the same motion". In the kinetic theory of gases such a lack of recognition appears in almost all of the modern mathematically oriented texts, for example, in [11], [19], [7], [6], [8] ([28] being a notable exception), and in all less mathematical and older texts. (I suspect this rather sloppy approach is largely due to the traditional philosophic viewpoint on the determinism of classical mechanics.)

In the specific case of the theory of existence and uniqueness for the Maxwell-Boltzmann equation proper, the situation happened to be in general better in this respect, simply because almost all results (see Section 1) were derived under the assumption $F \equiv 0$ in which case Axiom 2 is trivially satisfied. But the abovementioned lack of rigour can be noticed in papers dealing with existence-uniqueness questions for modified or somewhat generalized versions of the full Maxwell-Boltzmann equation (that is, roughly speaking, with $F \neq 0$ ). For example, this was the case in [23] and to a lesser extent in [9] and [10]. In [23] Povzner, considering a modified Naxwell-Boltzmann equation with a conservative $F$ independent of $t$ and $\boldsymbol{\xi}$, used a certain superposition of operations which is formally equivalent to a particular kind of the trajectorial shifter $\{\cdot\}_{\mathcal{B}}^{t}$, while assuming only that the $F^{\prime} s$ potential, $U$, belongs to $C(X)$. of course, this assumption was too weak even to guarantee local existence of solutions to the appropriate hamiltonian equations, and moreover, since Povzner's results for the modified Maxwell-Boltzmann equation were global on $T_{\infty} \times X \times \equiv$, even the essumption $U \in C^{2}(X)$ would not be sufficient to ensure the needed global fulfilment of Axiom 2. In [9] and [10], this author pointed out that his $F$ (defined almost identically to that of 
[23]) was assumed to be a continuous vector-function of $x$, thus Axiom 2 was valid at least locally. Unfortunately, under the influence of longstanding 'tradition', it was not explicitly stated in [9] and [10] that Axiom 2 is supposed valid in $R_{T}^{7}$ for a suitable $T$, although just this 'global' assumption was implicitly used in the papers in certain parts of the proofs. (The use of this assumption is apparent in the proof of Lemma 3.1 below.) While it would be sufficient to assume $F \in \operatorname{Lip}(X)$ uniformly, the assumption $F_{i} \in C^{1}(X) \quad(i=1,2,3), F \equiv\left(F_{1}, F_{2}, F_{3}\right)$, would not be sufficient since in particular the domain $R_{T}^{7}$ is unbounded; see also Remarks 2.1.

B. THE GENERAL INITIAL-VALUE PROBLEM

Assuming that Axioms 1 and 2 are satisfied on $R_{T}^{7}$ for every $t_{1}>t_{0}$ (unless otherwise stated), we shall first consider the following Cauchy problem closely related to the full conventional Maxwell-Boltzmann equation:

$$
D f=c(f, f), t>t_{0},
$$

with the initial condition

$$
f\left(t_{0}, x, \xi\right)=f_{0}(x, \xi) \geq 0,
$$

which on physical grounds should be such that at least

$$
\left(1+\xi^{2}\right) f_{0} \in L^{1}(\Xi)
$$

The quadratic operator $C(\cdot, \cdot)$ in (2.3) is formally given by

$$
\mathcal{C}(g, g) \equiv \int_{\Omega \times \Xi} V\left(g^{\prime} \hat{g}^{\prime}-g \hat{g}\right) d \omega d \hat{\xi},
$$

where

$$
\begin{aligned}
& \text { (2.5) })_{1} g \equiv g(t, \mathrm{x}, \xi), \hat{g} \equiv(t, \mathrm{x}, \hat{\xi}), g^{\prime} \equiv g\left(t, \mathrm{x}, \xi^{\prime}\right), \\
& \hat{g}^{\prime} \equiv g\left(t, x, \hat{\xi}^{\prime}\right), \\
& (2.5)_{2,3} \quad V \equiv \hat{\xi}-\xi, d \omega=r d r d \varepsilon,
\end{aligned}
$$


$(2.5)_{4,5}$

$$
\xi+\hat{\xi}=\xi^{\prime}+\hat{\xi}^{\prime}, \xi^{2}+\hat{\xi}^{2}=\xi^{\prime 2}+\hat{\xi}^{\prime 2} .
$$

(Hereafter, for brevity, we often use functional symbols to denote varying values of functions; also for any vector $\alpha, \alpha \equiv|\alpha|$.) The conventional Maxwell-Boltzmann equation is obtained from (2.3) on replacing the operator $D$ by the operator $D_{d}$ introduced in Remark 2.2.

In the usual terminology, $c(f, f)$ is the collision integral (which should be explicitly assumed (absolutely) convergent - a precaution which has often been neglected); $V$ is the velocity of a scattered molecule with respect to a scatterer; $r \geq 0$ and $\varepsilon \in[0,2 \pi)$, called respectively the impact parameter and the azimuthal angle, are geometric variables - polar coordinates in the impact parameter plane - of the elastic collision of two monatomic molecules, whose velocities $\xi$ and $\hat{\xi}$ before collision are for given $r$ and $\varepsilon$ uniquely transformed under the conservation laws $(2.5)_{4,5}$ to velocities $\xi^{\prime}$ and $\hat{\xi}^{\prime}$, respectively, after collision. (A detailed information about the linear mappings $(\xi, \hat{\xi}) \vec{\leftarrow}\left(\xi^{\prime}, \hat{\xi}^{\prime}\right)$ can be found in [11], Section 15, and [6], Chapter I, Section 7, Chapter II, Section 1.) The region $\Omega$ determines the limits of integration for the differential scattering cross-section $I(V, \theta)$ of spherically symetric scattering into a differential solid angle $(\theta, \theta+d \theta)$, where $\theta \in[0, \pi]$ is the colatitude (the polar axis is directed along $V$ ); by definition, $I(V, \theta) \sin \theta d \theta d \varepsilon=d \omega$. It is standard practice to assume the potential of (central) intermolecular force is repulsive; this guarantees in particular a 'safe' one-to-one passage from $\theta$ as a function of $r$ to its inverse (for example see [6], Chapter I, Section 7).

The volume of $\Omega, \int_{\Omega} d \omega$, is the so-called total collision crosssection. For an infinite range of intermolecular interaction, when $r \in[0, \infty)$ or $\theta \in[0, \pi]$, thevolume is clearly infinite; if the volume is finite we say that a cut-off (of the range of intermolecular interaction) has been applied. It should be mentioned that integration over $\Omega$ may, in some cases, be a priori artificial. This is the case with the pseudo-maxwellian molecular model. (For the original definition of this model, see [11], p. 244 and p. 247, Equation (20.4); a frequently used description of such molecules as simply cut-off maxwellian is ambiguous.) The same is true for a whole class of formal models which contains the 
pseudo-maxwellian as a particular case. (The existence of such a class of the so-called pseudomolecules [28] has been pointed out in [9].) The artificial integration is a specific feature of an implicit or unspecified cut-off defined later in this subsection.

For an alternative introduction of the collision integral, which is more abstract and somewhat less detailed from the physical point of view, see [23], [1], [26]; for other details of the collision integral, in particular for a thorough description of certain cut-offs, see [11], [19], $[7],[6]$; a thorough discussion of the (absolute) convergence of $\mathcal{C}(f, f)$ and related integrals is given in [28].

The value $t=t_{0}$ has been used above as the initial time, instead of $t=0$, to facilitate:

(i) a discussion of a continuation of the solution outside some 'basic' interval of its existence, and

(ii) a planned extension of this work to mixed problems for the full Maxwell-Boltzmann equation (compare with the " $F \equiv 0$ " case in [11], Section 19).

Also the last but not the least reason for the use of $t_{0}$ rather than $t=0$ is that, if the above axioms are satisfied for a particular given $F$ provided $t \in\left[t_{0}, t_{0}+\alpha\right]$ where $\alpha=$ const $>0$, they may not in general be satisfied for $t \in[0, \alpha]$.

Now, using a particular case of the trajectorial shifter along $\Gamma_{\infty}(t, x, \xi)$ (see Definitions 2.1 and 2.3), we may formally convert the Cauchy problem (2.3)-(2.5) into the problem of finding solutions of the following purely integral equation introduced in [9]:

$$
\begin{gathered}
f=A f, t>t_{0}, f_{0} \geq 0, \\
A f \equiv\left\{f_{0}\right\}_{t_{0}}^{t}+\int_{t_{0}}^{t}\{c(f, f)\}_{s}^{t} d s .
\end{gathered}
$$

Clearly this latter problem may or may not be equivalent to the earlier one or the conventional one, especially regarding smoothness properties of their solutions (see some comments below). Also note that, provided $\mathcal{C}(f, f)$ is convergent for $r \in[0, \infty)$, the equation $(2.6)$ is usually 
meaningful for molecules with infinite total collision cross-section.

Suppose that $\mathcal{C}(f, f)$ can be split into a difference of two terms, the creation-collision term $\mu(f, f)$ and the annihilation-collision term fuf , where the creation-collision operator $\mu(\cdot, \cdot)$ and the collision frequency operator $v(\cdot)$ are defined by the integrals

$$
\begin{aligned}
\mu(g, g) & \equiv \int_{\Omega \times \Xi} V g^{\prime} \hat{g}^{\prime} d \omega d \hat{\xi}, \\
v g & \equiv \int_{\Omega \times \Xi} V \hat{g} d \omega d \hat{\xi},
\end{aligned}
$$

(compare with (2.5)) which are both convergent although not necessarily bounded, for example if $\xi+\infty$. Then, using some trajectorial shifters of the same kind as in (2.6), we can formally rewrite the Cauchy problem (2.3)-(2.5) as the following integral equation ([10]):

$$
f=B f, t>t_{0}, f_{0} \geq 0
$$

$$
B f \equiv\left\{f_{\jmath}\right\}_{t_{0}}^{t} \exp \left[-\int_{t_{0}}^{t}\{v f\}_{s}^{t} d s\right]+\int_{t_{0}}^{t}\{\mu(f, f)\}_{u}^{t} \exp \left[-\int_{u}^{t}\{v f\}_{s}^{t} d s\right] d u
$$

The operator $B$ is in several respects more useful for the existence theory (including the theory for mixed problems) and its applications, than A . Specifically, in applications of certain direct and semi-direct schemes of successive approximations some of the advantages are:

(1) for a non-negative initial datum, $B$ leads through nonnegative approximations to a non-negative solution (this is not in general the case if one uses $A$ );

(2) the mapping condition, $B f \in \Phi$ if $f \in \Phi$, imposes usually weaker restrictions on the time interval involved than does an analogous condition for $A$;

(3) there is a definite indication in the available applications of reduced and/or modified versions of (2.9) that the first few approximations using $B$ give a much more precise picture of the flow of a Boltzmann gas than those obtained using A ;

(4) the so-called Knudsen iteration using the operator A fails 
altogether in the case of certain (one-dimensional) geometries. This fact was pointed out and partly explained by Willis (for example, in [32] and [33]). For a short explanation from a functional analysis view-point the reader is advised to consult Cercignani [6, pp. 136, 204].

The feature (3) is not really surprising, considering the physical interpretation of the equation (2.9). For example, see either Grad's discussion for $F \equiv 0$ in [11], Section 19, or a direct (that is omitting the conventional Maxwell-Boltzmann equation) physico-probabilistic construction of the reduced version of (2.9) for a general mixed problem in Vallander's paper [30], the ideas and terminology of which remind one of the so-called demographic method [21]. (It should be pointed out that [30] has some serious mathematical omissions discussed in detail elsewhere. Also Vallander's conclusion that the "Boltzmann kinetic equation is a decidedly impoverished consequence of our equations" seems to be very much exaggerated.)

Although as early as in 1958, Willis mentioned (for example, in [33]) the possibility of utilizing other integral forms of the reduced MaxwellBoltzmann equation, it was not until 1972 that this idea was applied by Arkeryd in his investigations of the initial-value problem for a generalized spatially homogeneous Maxwell-Boltzmann equation. The usefulness of such an idea in the case $F$ 丰 is still an open question.

Before proceeding further, we shall make some suggestions towards more precise terminology.

(S1) It is known (for example, see $[6$, p. 36]) that for the abovementioned splitting of $c(f, f)$ to be permissible, a cut-off of the intermolecular interaction has to be applied to eliminate contributions due to the so-called weak or grazing collisions which produce the troublesome divergencies. (From the point of view of the scattering process, $I(V, \theta)$ exhibits a nonintegrable singularity at the scattering angle $\theta=0$.) Suppose that such a cut-off is introduced without specific mention of any particular restriction(s) on the range of variables appearing in the definition of the differential scattering cross-section, and/or without reference to the kind of intermolecular interaction. (For example, a functional bound may be imposed on the kernel of the collision 
integral such that it is feasible only if the total collision cross-section is not infinite.) Then we may call such a cut-off unspecified or implicit, adding this terminology to that of radial cut-off and angular cut-off as introduced in 1967 by Cercignani (for details of those cut-offs, see [6], Chapter III). This gives us a terminology sufficiently complete to cover all cut-offs used in the existence theory of the Maxwell-Boltzmann equation. (Note that one may regard the application of any other cut-off, except the radial one, as a mathematical trick producing a kind of modified Maxwell-Boltzmann equation.)

(S2) Despite considerable usage of the phrase "classical solution of the Maxwell-Boltzmann equation" in recent literature, a specification of the term "classical" is lacking. To the best of this author's knowledge of the literature: a classical solution of the Maxwell-Boltzmann equation is an $L^{l}(\Xi)$-solution $\bar{f}$ of the equation $D_{d} f=\mathcal{C}(f, f)$, where $D_{d}$ is defined by $(2.2)_{2}$ in Remark 2.2, and so (according to Remarks 2.3) $\partial \bar{f} / \partial t$, $\partial \bar{f} / \partial x$, and $\partial \bar{f} / \partial \xi$ exist separately in some sense; an $L^{1}(\Xi)$-solution $\tilde{f}$ of the equation $0 f=C(f, f)$ should be called a generalized solution of the Maxwell-Boltzmann equation, unless it has been shown that $\tilde{f}$ is an $\bar{f}$. Naturally, one may object on historical, physical, and logical grounds to calling "classical" a solution $\bar{f}$ of $D_{d} f=c(f, f)$ which (say) attains some negative values, is such that $\overline{D f} \neq D_{d} \bar{f}$ (see Remark 2.4), and whose energy moment is divergent. In particular, to be a probability density function, $\bar{f}$ would have to be non-negative; also in all classical phenomenological derivations of the Maxwell-Boltzmanin equation, the motion of the test molecule is first considered along its free-streaming trajectory (that is, implicitly via $D$ ) and only then the operator $D$ is replaced by $D_{d}$. Hence, for evident reasons, we propose the name traditional solution for a non-negative classical solution $\bar{f}$, satisfying (2.2) $)_{I}$ with $D_{d} \bar{f}$ existing at least almost everywhere, and such that $(1+\xi)^{3} \bar{f} \in L^{1}(\equiv)$.

Now, by slightly extending Wild's remark in [31] on the reduced version of (2.9), one can statethat differentiable solutions of (2.6) or (2.9) are also classical solutions of the conventional version of the Cauchy problem (2.3)-(2.5) or, under some obvious assumptions, traditional 
solutions of (2.3)-(2.5). In the general case, however, it is not known whether a solution, $\tilde{f}$, of $(2.6)$ or $(2.9)$ has to be classical. While it can usually be rather simply shown that $\tilde{D f}$ is defined in an appropriate sense on the time interval of the existence of $\tilde{f}$ and there satisfies suitable conditions (as an illustration of this, see the proof of Theorem 3.2 below), the mere existence, in one sense or another, of the separate derivatives $\partial \tilde{f} / \partial t, \partial \tilde{f} / \partial x$, and $\partial \tilde{f} / \partial \xi$ seems to require a number of additional smoothness-boundedness restrictions to be imposed on certain of the (distribution) functions involved (see examples of this in Grad's papers [14] and [15]).

It is not yet clear to this author whether the recent approach of Shizuta and others [27], [26], leading to some types of classical solutions for the reduced version of the Maxwell-Boltzmann equation with angularly cut-off hard potential, is appropriate in the general case under consideration, since that case involves non-trivial trajectorial shifters. Also the passage from potentials with angular cut-offs to potentials with radial cut-offs appears to be a subtle and insufficiently clarified matter. However, in Shizuta's opinion $[26, \S 8]$ his method should be applicable to the standard mixed problem in the case of the radially cut-off hard potentials and reduced Maxwell-Boltzmann equation. (No proof of this conjecture was available in [26], but it looks very sound, in particular, because the angularly cut-off hard potentials were intentionally introduced by Grad as an 'analogy' to the billiard-ball molecular model [13, p. 31].)

We end this section by recalling that in the case when (2.6) is used (say, as the basis for successive approximations), there is additional difficulty in showing that the solution obtained (classical or generalized) is non-negative.

\section{A particular generalized initial-value problem}

A. FORMULATION OF THE PROBLEM

Let $\left(a_{0}, b, c, q, U_{*}\right)$ be an arbitrary fixed point of $R_{+}^{5}$ where $R_{+} \equiv(0, \infty)$.

Henceforth we shall only consider the particular case of the initialvalue problem (2.3)-(2.5) specified by the following assumptions: 
(3.1) Axioms 1 and 2 (as given in Subsection 2.A) are valid in $R_{T}^{7}$ with $T=\left[t_{0}, t_{0}+q^{-1}\right]$ under the assumption that (for all $x \in X)$

(3.1) $F=-\frac{\partial U}{\partial x}$, where $U$ is a sufficiently smooth function of $x$ alone such that

$$
\begin{aligned}
& \inf U=u_{*} ; \\
& \int_{\Omega} d \omega \leq b ; \\
& f_{0} \in C(x \times \Xi), \\
& 0 \leq f_{0} e^{Q} \leq a_{0},
\end{aligned}
$$

where

$$
Q \equiv c\left(\xi^{2}+2 U\right)
$$

that is, $Q$ is proportional to the total mechanical energy of the test molecule free-streaming in the field $F$.

\section{B. MAIN RESULTS}

In what follows we denote by $P(X)$ the set of functions $F$ satisfying the assumptions $(3.1)_{1,2}$ and such that Axiom 2 is valid on $R_{T}^{7}$ with $T$ as given in (3.1). The set of functions $f_{0}$ satisfying (3.3)-(3.5) will be denoted by $\Phi_{0}(X \times \Xi)$.

In addition, we assume in the following that,

(3.6). unless otherwise stated, the variable $t$ is restricted to the interval $\tau_{\beta} \equiv\left[t_{0}, t_{0}+\beta\right]$, where $\beta=$ const $\in\left(0, q^{-1}\right)$, and then introduce the scalar expressions $\psi$ and $\bar{Q}$ defined by (3.7)

$$
\psi \equiv \psi(t) \equiv c\left(1-q\left(t-t_{0}\right)\right),
$$


The Maxwell-Boltzmann equation

399

$$
\bar{Q} \equiv\left(1-q\left(t-t_{0}\right)\right) Q \equiv\left(\xi^{2}+2 U\right) \psi
$$

of course, in view of $(3.6),(3.5)$, and $(3.1)_{2}$,

$$
\text { (3.9) } \inf _{t \in \tau_{B}} \psi>0, \inf _{(x, \xi)} Q>0 \text {, and } \inf _{(t, x, \xi) \in R_{\tau_{B}}^{7}} \bar{Q}>0 \text {. }
$$

For each $\alpha \in(0, B]$, we denote by $\Phi_{\alpha}\left(R_{\tau}^{7}\right)$ the Banach space of functions $f$ such that

$$
\begin{gathered}
f \in C\left(R_{\tau}^{7}\right), \\
\sup _{(t, \mathrm{x}, \xi) \in R_{\tau}^{7}}\left(|f| e^{\bar{Q}}\right)<\infty,
\end{gathered}
$$$$
(3.10)_{2}
$$

where

$$
\tau \equiv\left[t_{0}, t_{0}+\alpha\right],
$$

with the basic norm, $\|\cdot\|_{\Phi_{\alpha}}$, given by

$$
\quad\|f\|_{\Phi_{\alpha}}=\sup _{(t, x, \xi) \in R_{\tau}^{7}}\left(|f| e^{\bar{Q}}\right) .
$$

$$
\begin{aligned}
& \text { In every } \Phi_{\alpha}\left(R_{\tau}^{T}\right) \text { we also introduce a certain class of norms, } \\
& \|\cdot\|_{\Phi_{\alpha} ; p} \text {, equivalent to }\|\cdot\|_{\Phi_{\alpha}} \text { and defined by } \\
& \qquad \begin{aligned}
\| .11) \quad & \sup _{\Phi_{\alpha} ; p}\left(|f| e^{\bar{Q}-p\left(t-t_{0}\right)}\right), \\
p & =\text { canst } \in R_{+} .
\end{aligned}
\end{aligned}
$$

The equivalence of the norms follows immediately from

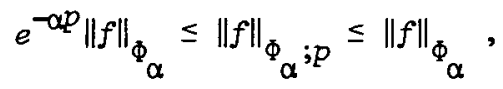

for all $(\alpha, p, f) \in(0, \beta] \times R_{+} \times \Phi_{\alpha}\left(R_{\tau}^{7}\right)$. (One needs to check

https://doi.org/10.1017/S0004972700023480 Published online by Cambridge University Press 
the equivalence since each $\Phi_{\alpha}\left(R_{\tau}^{7}\right)$ is infinite-dimensional. For a finitedimensional space all norms are equivalent.)

Let $\Phi_{\alpha, 1}\left(R_{\tau}^{7}\right)$ be the closed subset of $\Phi_{\alpha}\left(R_{\tau}^{7}\right)$ such that $f \in \Phi_{\alpha, \perp}\left(R_{\tau}^{7}\right) \quad$ if

$$
\begin{gathered}
f \geq 0, \\
\|f\|_{\phi_{\alpha} \leq a_{1},} a_{1}=\text { const }>0 .
\end{gathered}
$$

Clearly $\Phi_{\alpha, l}\left(R_{\tau}^{7}\right)$ is not a Banach space, as it is not closed under addition, but it is a complete metric space under the metric induced by any of the norms $\|\cdot\|_{\phi_{\alpha} ; p}, p \in[0, \infty)$.

REMARK. Note that the set $\bar{C}_{0}^{+}$in Theorem 3 (a) of [10] should be understood as $\Phi_{\alpha, 1}\left(R_{\tau}^{7}\right)$ with $\alpha$ restricted to a certain range. It is proper to mention here that some sloppiness in the introduction of the notation ${\underset{0}{\mathrm{R}}}_{0}$ in [9] (see also [10], Section 2) resulted in subsequent sloppiness in some parts of [9] and [10]. Since appropriate corrections of [9] and [10] are simple and can be easily deduced either directly from those papers, or by comparison with the present one, we shall not elaborate upon them separately.

REMARK 3.1. In the proofs of the lemmas and theorems of this section we shall frequently use the fact that

(3.13) any function of $Q$ alone is invariant under the operation $\{\cdot\}_{s}^{t}$ (where $\{\cdot\}_{s}^{t}$ is the trajectorial shifter introduced in Definitions 2.3).

This property can be immediately deduced from the comment following the definition (3.5).

The following lemma, to be used later in our investigations, represents an improvement of a result contained in [10]. (We remind the reader that in this section we restrict our attention to the strengthening 
of the results of [10].) Since appropriate parts of the proof of the result as given in that paper are too condensed, and since some estimates needed for the lemma are scattered throughout [9] and [10], the basic steps of the proof of the lemma are given below. (Note changes in the notation compared with [9] and [10].)

LEMMA 3.1. Given

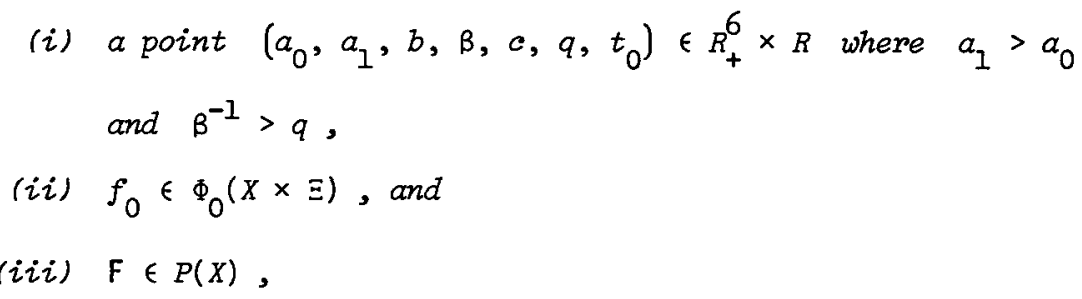

there is a largest constant $\gamma \in(0, \beta]$ such that, for any $\alpha \in(0, \gamma]$ and $\int_{\Omega} d \omega \leq b, B \Phi_{\alpha, 1}\left(R_{\tau}^{7}\right) \subseteq \Phi_{\alpha, 1}\left(R_{\tau}^{7}\right)$ for the operator $B$ as defined in (2.9). The constant $\gamma$ satisfies the inequality

$$
\gamma \geq t^{*}-t_{0}
$$

where $t^{*}$ is the largest constant from the interval $\left(t_{0}, t_{0}+\beta\right]$ such that

$$
\sup _{Q \in\left[2 c U_{*}, \infty\right)} n\left(t^{*}, Q\right) \leq \frac{1}{\pi a_{1}^{2} b}\left(a_{1}-a_{0}\right) .
$$

Here

$$
\eta(t, Q) \equiv e^{-2 \psi U_{*}-2} \phi\left(\psi^{\frac{1}{2}} \xi^{*}\right) \frac{1-e^{-q\left(t-t_{0}\right) Q}}{q Q},
$$

where

$$
\begin{gathered}
\phi(y) \equiv 2+\pi^{\frac{1}{2}} y, \\
\xi^{*} \equiv \sqrt{(Q / c)-2 U_{*}} .
\end{gathered}
$$

REMARK. An easy proof that such a constant $t^{*}$ does exist is left to the reader.

REMARKS 3.2. We draw attention to the importance of the properties (3.9) in the proofs of this section. Henceforth, to avoid repetition, direct references to (3.9) will usually be omitted. For brevity, we shall 
also omit direct references to the definitions given in Subsection 2.A.

Proof of Lemma 3.1. In view of (2.7)-(2.9), (3.4), (3.10) 4 , (3.12) 1,2 , the property (3.13) and other (more elementary) properties of the trajectorial shifter, we may write (for all $t \in \tau_{\beta}$ )

$$
\begin{aligned}
0 \leq B f & \leq\left\{f_{0}\right\}_{t_{0}}^{t}+\int_{t_{0}}^{t}\{\mu(f, f)\}_{u}^{t} d u \\
& \leq a_{0} e^{-Q}+a_{1}^{2} \int_{t_{0}}^{t}\left\{\mu\left(e^{-\bar{Q}}, e^{-\bar{Q}}\right)\right\}_{u}^{t} d u
\end{aligned}
$$

Since, by the conservation law $(2.5)_{5}$ and the definitions (2.7) and (2.8), we have

$$
\mu\left(e^{-\bar{Q}}, e^{-\bar{Q}}\right)=e^{-\bar{Q}} \nu\left(e^{-\bar{Q}}\right),
$$

the preceding inequality can be replaced by:

$$
0 \leq B f \leq a_{0} e^{-Q}+a_{1}^{2} \int_{t_{0}}^{t}\left\{e^{-\bar{Q}} \nu\left(e^{-\bar{Q}}\right)\right\}_{u}^{t} d u
$$

for all $t \in \tau_{\beta}$.

Using the assumption (3.2), the estimate $V \leq \xi+\hat{\xi} \quad\left(\right.$ see $\left.(2.5)_{2}\right)$, the definition (3.8), and the property (3.13), we can show that

$$
\text { (3.18) } \begin{aligned}
& \int_{t_{0}}^{t}\left\{e^{-\bar{Q}} v\left(e^{-\bar{Q}}\right)\right\}_{u}^{t} d u \leq \pi b \int_{t_{0}}^{t}\left\{e^{-(\bar{Q}+2 \psi U)} \psi^{-2} \phi\left(\psi^{\frac{3}{2}} \xi\right)\right\}_{u}^{t} d u \\
& \leq \pi b e^{-Q} \int_{t_{0}}^{t} e^{q\left(u-t_{0}\right) Q-2 \psi(u) U_{*}}[\psi(u)]^{-2} \times \\
& \times \phi(\xi * \sqrt{\psi(u)}) d u,
\end{aligned}
$$

where $U_{*}, \phi$, and $\xi^{*}$ are defined by $(3.1)_{2},(3.15)_{2}$, and $(3.15)_{3}$, respectively. Hence, by (3.8) and (3.18), (3.17) implies 
(3.19) $0 \leq e^{\bar{Q}_{B f}} \leq e^{-q\left(t-t_{0}\right) Q} \times$

$$
\times\left(a_{0}+\pi a_{1}^{2} b \int_{t_{0}}^{t} e^{q\left(u-t_{0}\right) Q-2 \psi(u) U_{*}}[\psi(u)]^{-2} \phi(\xi * \sqrt{\psi(u)}) d u\right)
$$

Let us now restrict attention to times $t$ in the interval $\left[t_{0}, t^{*}\right]$, where $t^{*}$ is as specified above in the statement of the lemma, and denote $\psi\left(t^{*}\right)$ by $\psi_{*}$. Then (for all $t \in\left[t_{0}, t^{*}\right]$ ) we have, using in particular

(i) the evident monotonicity with respect to $t$ of some functions on the right-hand side of (3.19) and

(ii) the definition $(3.15)_{1}$,

$$
\begin{aligned}
& 0 \leq e^{\bar{Q}_{B f}} \leq a_{0}+\pi a_{1}^{2} b e^{-q\left(t-t_{0}\right) Q} \psi_{*}^{-2} \phi\left(\psi_{*}^{\frac{3}{2}} \xi^{*}\right) e^{-2 \psi_{*} U_{*}} \frac{e^{q\left(t-t_{0}\right) Q}-1}{q Q} \\
& \leq a_{0}+\pi a_{1}^{2} b \psi_{*}^{-2} \phi\left(\psi_{*}^{\frac{3}{2}} \xi^{*}\right) e^{-2 \psi_{*} U_{*}} \frac{1-e^{-q\left(t^{*}-t_{0}\right) Q}}{q Q} \\
& =a_{0}+\pi a_{1}^{2} b n\left(t^{*}, Q\right) \text {. }
\end{aligned}
$$

Therefore by (3.15),

$$
0 \leq e^{\bar{Q}_{B f}} \leq a_{1}
$$

for all $f \in \Phi_{\alpha, 1}\left(1^{7} \tau\right)$ and $\alpha \in\left(0, t^{*}-t_{0}\right]$.

To complete the proof of the lemna, we shall show that $B f$ is continuous for every $f \in \Phi_{\alpha, 1}\left(R_{\tau}^{7}\right)$, where $\alpha \in(0, B]$. We notice first that it is sufficient if we prove that $\nu f$ and $\mu(f, f)$ are continuous functions for any such $f$. This follows by using assumptions (3.1) and (3.6) in association with Lemmas 2.1 and 2.2 , and by referring to the definitions of the operators $\nu, \mu$, and $B$ (see (2.7)-(2.9)); also note (3.3).

We shall only outline the proof of the continuity of $v f$, as it will be seen that the proof for $\mu(f, f)$ is similar, in view of $(3.12)_{2}$ and (3.16). Now, given $\xi \in \Xi$, we choose an open ball $B$ in $\Xi$, with centre 
at the origin of $\Xi$ and such that $\xi \in B$, and we define the function $g$ on the set $\tau_{B} \times X \times B \times \Xi$ by the identity $g(t, x, \xi, \xi) \equiv V f(t, x, \hat{\xi})$. Then, using the inequality $V \leq \xi+\hat{\xi}$ and the assumption (3.12) 2 , we obtain a functional bound for $g$ which depends only on $\hat{\xi}$ (strictly speaking, only on $\hat{\xi}$ ) and is integrable on $\Xi$. On the basis of this and some other (more evident) properties of $g$, we can deduce the required continuity of $v f$ by applying the Lebesgue dominated convergence theorem.

The lemma now follows from the preceding considerations on taking into account the nature of the above estimates which lead to (3.21).

REMARK 3.3. The above proof of the continuity of $B f$ is of course valid for every $f \in \Phi_{\alpha}\left(R_{\tau}^{7}\right)$ - a fact which is used in a forthcoming paper.

It has been proved in [10] that the operator $B$ satisfies in terms of the 'basic' metric induced by $\|\cdot\|_{\Phi}$ a Lipschitz condition on $\Phi_{\alpha, 1}\left(R_{\tau}^{T}\right)$ for each $\alpha \in(0, B]$, but to show that $B$ is a contraction on $\Phi_{\alpha, 1}\left(R_{\tau}^{7}\right)$, it is additionally assumed in [10] that $\alpha$ is sufficiently small. In Theorem 3.1 below we present a mach stronger result which is mainly due to the introduction in $\Phi_{\alpha}\left(R_{\tau}^{T}\right)$ of the class of equivalent norms $\|\cdot\|_{\Phi} ; p$, $p \in R_{+}$, defined by (3.11). For convenience, we denote by $\Phi_{\alpha, 1 ; p}$ the complete metric space which consists of the set $\Phi_{\alpha, 1}\left(R_{\tau}^{7}\right)$ equipped with the metric induced by the equivalent norm $\|\cdot\|_{\Phi_{\alpha} ; p}$.

REMARK 3.4. It is of basic importance for what follows to realise that the introduction of the equivalent norms (3.11) does not affect $\Phi_{\alpha, 1}\left(R_{\tau}^{7}\right)$. Therefore we can be free in our selection of a suitable $p$, or a suitable range for $p$.

THEOREM 3.1. If

(i) $\left(a_{0}, a_{1}, b, \beta, c, q, t_{0}\right)$ is a point of $R_{+}^{6} \times R$ such that $B^{-1}>q$, 
(ii) $f_{0} \in \Phi_{0}(X \times \equiv)$, and

(iii) $\mathrm{F} \in P(X)$,

then for each $\alpha \in(0, B]$ there exists a constont $p_{\alpha} \in R_{+}$such that the operator $B$ is a contraction on $\Phi_{\alpha, 1 ; p}$ for any $p>p_{\alpha}$, provided

$$
\text { (iv) } \int_{\Omega} d \omega \leq b \text {. }
$$

Proof. For any $f_{1}, f_{2} \in \Phi_{\alpha, 1}\left(R_{\tau}^{T}\right)$ where $\alpha \in(0, \beta]$, we have (see [10], Section 3),

(3.22) $\left|B f_{1}-B f_{2}\right| \leq a_{0} e^{-Q} \int_{t_{0}}^{t}\left\{v\left|f_{1}-f_{2}\right|\right\}_{s}^{t} d s+$

$$
\begin{aligned}
& +\int_{t_{0}}^{t}\left\{\left|\mu\left(f_{1}, f_{1}\right)-\mu\left(f_{2}, f_{2}\right)\right|\right\}_{u}^{t} d u+ \\
& +\int_{t_{0}}^{t}\left\{\mu\left(f_{1}, f_{1}\right)\right\}_{u}^{t} d u \int_{t_{0}}^{t}\left\{v\left|f_{1}-f_{2}\right|\right\}_{s}^{t} d s .
\end{aligned}
$$

Denote successive terms on the right-hand side of (3.22) by $K_{1}\left(f_{1}, f_{2}\right), K_{2}\left(f_{1}, f_{2}\right)$, and $K_{3}\left(f_{1}, f_{2}\right)$; then using the definition (3.11) as well as some further manipulations and estimates similar to those used in [9], [10], and in the proof of Lemma 3.1, we may write for any $\alpha \in(O, \beta]$ the following estimates :

$$
\begin{aligned}
K_{1}\left(f_{1}, f_{2}\right) & \leq a_{0} e^{-Q}\left\|f_{1}-f_{2}\right\|_{\Phi_{\alpha} ; p} \int_{t_{0}}^{t}\left\{v\left(e^{-\bar{Q})} e^{p\left(t-t_{0}\right)}\right\}_{s}^{t} d s\right. \\
& \leq \pi a_{0} b e^{-\left(Q+2 \psi_{\alpha} U_{*}\right)} \psi_{\alpha}^{-2} \phi\left(\psi_{\alpha}^{\frac{1}{2}} \xi^{*}\right) \|_{f_{1}-f_{2} \|_{\Phi_{\alpha}} ; p} \int_{t_{0}}^{t} e^{p\left(s-t_{0}\right)} d s \\
& \equiv M_{1}(t, Q ; p) e^{-\bar{Q}+p\left(t-t_{0}\right)}\left\|f_{1}-f_{2}\right\|_{\Phi_{\alpha} ; p},
\end{aligned}
$$

where

$$
\psi_{\alpha} \equiv \inf _{t \in \tau} \psi=\psi\left(t_{0}+\alpha\right)
$$


406

Aleksander GIikson

and

(3.25) $M_{1}(t, Q ; p) \equiv \pi a_{0} b e^{-\left(2 \psi_{\alpha} U_{*}+Q q\left(t-t_{0}\right)\right)} \psi_{\alpha}^{-2} \phi\left(\psi_{\alpha^{\frac{2}{2}} \xi^{*}}\right) \frac{1-e^{-p\left(t-t_{0}\right)}}{p}$;

(3.26) $k_{2}\left(f_{1}, f_{2}\right) \leq 2 a_{1}\left\|f_{1}-f_{2}\right\|_{\Phi_{\alpha}} ; p \int_{t_{0}}^{t}\left\{e^{-\bar{Q}} v\left(e^{-\bar{Q})} e^{p\left(t-t_{0}\right)}\right\}_{u}^{t} d u\right.$

$$
\begin{aligned}
& \leq 2 \pi a_{1} b e^{-\left(Q+2 \psi_{\alpha} U_{*}\right)} \psi_{\alpha}^{-2 \phi\left(\psi_{\alpha}^{\frac{1}{2}} \xi^{*}\right)\left\|f_{1}-f_{2}\right\|_{\Phi_{\alpha}} ; p} \times \\
& \times \int_{t_{0}}^{t} e^{(q Q+p)\left(u-t_{0}\right)} d u \\
& \equiv M_{2}(t, Q ; p) e^{-\bar{Q}+p\left(t-t_{0}\right)}\left\|f_{1}-f_{2}\right\|_{\Phi_{\alpha} ; p},
\end{aligned}
$$

where

$(3.27)$

$$
M_{2}(t, Q ; p) \equiv 2 \pi a_{1} b e^{-2 \psi_{\alpha} U_{*}} \psi_{\alpha}^{-2} \phi\left(\psi_{\alpha}^{\frac{2}{2}} \xi^{*}\right) \frac{1-e^{-(Q q+p)\left(t-t_{0}\right)}}{Q q+p}
$$

and, using the estimate of $\int_{t_{0}}^{t}\left\{v\left|f_{1}-f_{2}\right|\right\}_{s}^{t} d s$ available in (3.23),

(3.28) $K_{3}\left(f_{1}, f_{2}\right) \leq \frac{a_{1}^{2}}{a_{0}} e^{Q} M_{1}(t, Q ; p) e^{-\bar{Q}+p\left(t-t_{0}\right)}\left\|f_{1}-f_{2}\right\|_{\Phi} ; p \times$

$$
\times \int_{t_{0}}^{t}\left\{e^{-\bar{Q}} v\left(e^{-\bar{Q}}\right)\right\}_{u}^{t} d u
$$

$$
\equiv M_{3}(t, Q ; p) e^{-\bar{Q}+p\left(t-t_{0}\right)}\left\|f_{1}-f_{2}\right\|_{\Phi} ; p,
$$

where

$$
M_{3}(t, Q ; p) \equiv \frac{a_{1}^{2}}{a_{0}} M_{1}(t, Q ; p) e^{Q} \int_{t_{0}}^{t}\left\{e^{-\bar{Q}} v\left(e^{-\bar{Q}}\right)\right\}_{u}^{t} d u
$$

Now, by $(3.5),(3.9),(3.15)_{2,3},(3.24)$, and the estimate

https://doi.org/10.1017/S0004972700023480 Published online by Cambridge University Press 


$$
\int_{t_{0}}^{t}\left\{e^{-\bar{Q}} v\left(e^{-\bar{Q}}\right)\right\}_{u}^{t} d u \leq \pi b e^{-\left(Q+2 \psi_{\alpha} U_{*}\right)} \psi_{\alpha}^{-2} \phi\left(\psi_{\alpha}^{\frac{2}{2}} \xi^{*}\right) \frac{e^{q\left(t-t_{0}\right) Q}-1}{q Q}
$$

(which is evident from the estimates $(3.17)-(3.20))$, it follows from (3.25), (3.27), and (3.29) that $M_{i}(\cdot, \cdot, \cdot), i=1,2,3$, are bounded functions on $\mathrm{T} \times\left[20 U_{*}, \infty\right) \times R_{+}$. Subsequently, using the estimates (3.23), (3.26), (3.28), and the definition (3.11) (on taking into account the continuity of $B f$ for $f \in \Phi_{\alpha, 1}\left(R_{T}^{7}\right)$ with $\alpha \in(0, B]$ as shown in the proof of Lemma 3.1), we obtain from (3.22),

$$
\left\|B f_{1}-B f_{2}\right\|_{\Phi_{\alpha} ; p} \leq \varepsilon_{\alpha}(p)\left\|f_{1}-f_{2}\right\|_{\Phi_{\alpha} ; p},
$$

where

$$
\varepsilon_{\alpha}(p) \equiv \sum_{i=1}^{3} M_{i \alpha}^{*}(p)
$$

$(3.31)_{1} M_{i \alpha}^{*}(p) \equiv \sup _{(t, Q) \in \tau \times\left[2 c U_{*}, \infty\right)} M_{i}(t, Q ; p), i=1,2,3$,

$M_{i \alpha}^{*}(p)$ finite (and greater than 0 ) for all $(\alpha, p) \in(0, \beta] \times R_{+}$.

$$
\text { Moreover, }
$$

$$
M_{i \alpha}^{*}(y) \rightarrow 0 \text { for } y+\infty, \quad i=1,2,3 \text {. }
$$

Hence, by $(3.31)-(3.32)$,

(3.33) for each $\alpha \in(0, B]$, there exists

$$
p_{\alpha} \in R_{+} \mid\left(p \in\left(p_{\alpha}, \infty\right) \Rightarrow 0<\varepsilon_{\alpha}(p)<1\right) \text {. }
$$

I'his completes the proof.

REMARK. As is seen from its proof, Theorem 3.1 can be replaced by a more general one if needed.

REMARK 3.5. Clearly, the abstract Lipschitz condition (3.30) remains valid for $p=0$; that is, for the basic norm $\|\cdot\|_{\Phi_{\alpha}}$ and with the Lipschitz constant $\varepsilon_{\alpha}\left(0^{+}\right)$.

The following lemma will help to establish property (iv) in Theorem 
3.2 below. (We remind the reader that the operator $B$ is defined in (2.9).) For brevity in the statement of the lemma $\varepsilon_{\alpha}(0)$ is taken to denote $\varepsilon_{\alpha}\left(0^{+}\right)$.

LEMMA 3.2. If $B(\cdot, \cdot)$ denotes the operator defined by

$$
B\left(f_{0}, f\right) \equiv B f,
$$

then, under the assumptions (i), (iii), and (iv) of Theorem 3.1,

$$
\left\|B\left(f_{0}, f\right)-B\left(g_{0}, g\right)\right\|_{\Phi_{\alpha} ; p} \leq\left\|f_{0}-g_{0}\right\|_{\Phi_{\alpha} ; p}+\varepsilon_{\alpha}(p)\|f-g\|_{\Phi_{\alpha} ; p}
$$

for any $f_{0}, g_{0} \in \Phi_{0}(X \times \Xi)$, any $f, g \in \Phi_{\alpha, I}\left(R_{\tau}^{7}\right)$, and any $p \in[0, \infty)$.

Proof. We begin from the following estimate which is a 'generalization' of (3.22):

$$
\left|B\left(f_{0}, f\right)-B\left(g_{0}, g\right)\right| \leq K\left(f_{0}, g_{0}, f, g\right)+K_{2}(f, g)+K_{3}(f, g),
$$

where

(3.36) $k\left(f_{0}, g_{0}, f, g\right) \equiv$

$$
\equiv\left|\left\{f_{0}\right\}_{t_{0}}^{t} \exp \left[-\int_{t_{0}}^{t}\{v f\}_{s}^{t} d s\right]-\left\{g_{0}\right\}_{t_{0}}^{t} \exp \left[-\int_{t_{0}}^{t}\{v g\}_{s}^{t} d s\right]\right|,
$$

and where $K_{2}(\cdot, \cdot)$ and $K_{3}(\cdot, \cdot)$ are as defined in the first statement after $(3.22)$.

Applying now to $K\left(f_{0}, g_{0}, f, g\right)$ the inequality

$$
\left|k_{1} k_{2}-k_{3} k_{4}\right| \leq\left|k_{1}\left(k_{2}-k_{4}\right)\right|+\left|k_{4}\left(k_{1}-k_{3}\right)\right| \text {, }
$$

and next using

(i) the bound (3.4),

(ii) the estimate

$$
\left|\exp \left[-\int_{t_{0}}^{t}\{v f\}_{s}^{t} d s\right]-\exp \left[-\int_{t_{0}}^{t}\{v g\}_{s}^{t} d s\right]\right| \leq \int_{t_{0}}^{t}\{v|f-g|\}_{s}^{t} d s,
$$

and 
(iii) some simple properties of the trajectorial shifter, we obtain from (3.36)

$$
K\left(f_{0}, g_{0}, f, g\right) \leq K_{1}(f, g)+\left\{\left|f_{0}-g_{0}\right|\right\}_{t_{0}}^{t}
$$

Since $f_{0}, g_{0} \in \Phi_{0}(X \times \Xi)$ and $\left\{e^{(Q q+p)\left(t-t_{0}\right)}\right\}_{t_{0}}^{t}=1$, we have on the basis of (3.8), (3.11), and the property (3.13),

$$
\begin{aligned}
\left\{\left|f_{0}-g_{0}\right|\right\}_{t_{0}}^{t} & \leq e^{-Q}\left\|f_{0}-g_{0}\right\|_{\Phi_{\alpha} ; p} \\
& \leq e^{-\bar{Q}+p\left(t-t_{0}\right)}\left\|f_{0}-g_{0}\right\|_{\Phi_{\alpha} ; p}
\end{aligned}
$$

for all $p \in[0, \infty)$.

The result to be proved follows easily from

(i) $(3.35)-(3.38)$,

(ii) those estimates in the proof of Theorem 3.1 which lead to $(3.30)$, and

(iii) Remark 3.5 .

We are now ready to prove the principal result of this section.

THEOREM 3.2. Let $\left(a_{0}, a_{1}, b, \beta, c, q, t_{0}\right)$ be a point of $R_{+}^{6} \times R$ such that $a_{1}>a_{0}$ and $B^{-1}>q$, and let $F$ belong to $P(X)$. Then for each $\alpha \in(0, \gamma]$, where $\gamma$ is as specified in Lemm 3.1, the Cauchy problem (2.3)-(2.5) with an arbitrary $f_{0} \in \Phi_{0}(X \times \Xi)$, and under the 'cutoff' assumption (3.2), has in $\Phi_{\alpha, 1}\left(R_{\tau}^{T}\right)$ a unique solution $f \equiv \tilde{f}$. Specifically (for each $\alpha \in(0, \gamma]$ ):

(i) $\tilde{f}$ is differentiable along the $R_{\tau}^{\top}$-trajectories;

(ii) $\tilde{D f}$ and $\mathcal{C}(\tilde{f}, \tilde{f})$ are identical bounded continuous functions vanishing for $\xi \rightarrow \infty$;

(iii) $\tilde{f}=\lim _{n \rightarrow \infty} f_{n}$, where $f_{n+1}=B f_{n}(n=1,2, \ldots)$, and $f_{1}$ 
is an arbitrary element of $\Phi_{\alpha, 1}\left(R_{\tau}^{7}\right)$; there exists a number $p_{\alpha} \in R_{+}$such that for any $p>p_{\alpha}$ the error estimate of the successive approximations may be given by

$$
\left\|f_{n+1}-\tilde{f}\right\|_{\Phi_{\alpha} ; p} \leq\left(\varepsilon_{\alpha}(p)\right)^{n}\left(1-\varepsilon_{\alpha}(p)\right)^{-1}\left\|f_{1}-f_{2}\right\|_{\Phi_{\alpha} ; p} \text {, }
$$

where $\varepsilon_{\alpha}(p) \in(0,1)$ and is defined by $(3.31),(3.31)_{1}$;

(iv) for any number $p>\inf \left\{p_{\alpha}\right\}, \tilde{f}$ satisfies an abstract Lipschitz condition on $\Phi_{0}(X \times \Xi)$ for the norm $\|\cdot\|_{\Phi_{\alpha} ; p}$ with a Lipschitz constant greater than or equal to $\left(1-\varepsilon_{\alpha}(p)\right)^{-1}$ uniformly with respect to the set $\left\{\left(t_{0}, t\right) \mid t_{0} \in R ; t \in \tau\right\} ;$ in particular, within $\Phi_{0}(X \times \equiv)$ the solution $\tilde{f}$ depends continuously on $f_{0}$ with respect to any norm $\|\cdot\|_{\Phi_{\alpha} ; p}$ where $p>\inf \left\{p_{\alpha}\right\}$.

Proof. On the basis of Lemma 3.1, Theorem 3.1, and the principle of contracting mappings, it immediately follows that the operator $B$ has, for each $\alpha \in(0, \gamma]$, a unique fixed point $f \equiv \tilde{f}$ in $\Phi_{\alpha, 1}\left(R_{\tau}^{T}\right)$. Applying the operator $D$ (for example, via Remark 2.6) to both sides of the identity $\tilde{f} \equiv \hat{B f}$, and using in particular Lemma 2.2 and Lemma 2.3, we establish the existence of the total derivative $0 \tilde{f}$ by establishing the differentiability along the $R_{\tau}^{7}$-trajectories of various factors and terms appearing in the expansion of $D B \tilde{f}$. Moreover, we ultimately obtain that $D B \tilde{f}=-\tilde{f} \cup \tilde{f}+\mu(\tilde{f}, \tilde{f}) \equiv \mathcal{C}(\tilde{f}, \tilde{f})$. In such a way we arrive at the conclusion that $\tilde{f}$ satisfies the Cauchy problem (2.3)-(2.5). More precisely, since we have already shown in the proof of Lemma 3.1 that for any $f \in \Phi_{\alpha, \perp}\left(R_{\tau}^{7}\right)$ both $f \cup f$ and $\mu(f, f)$ are non-negative continuous functions bounded by $\pi a_{1}^{2} b e^{-\left(\bar{Q}+2 \psi_{\alpha} U_{*}\right)} \psi_{\alpha}^{-2} \phi\left(\psi_{\alpha}^{\frac{1}{2}} \xi^{*}\right)$, where $a_{1}, b, \psi_{\alpha}, U_{*}$ are positive numbers and $\xi^{*}, \phi$ are defined by $(3.15)_{2,3}$, the solution $\tilde{f}$ has the property (ii). 
The statement (iii) is obviously an appropriate (taking account of (3.30)-(3.33)) version of a standard statement contained in the principle of contracting mappings.

To prove (iv) we consider two solutions, $\tilde{f}$ and $\tilde{g}$ say, of the Cauchy problem (2.3)-(2.5) in $\Phi_{\alpha, 1}\left(R_{\tau}^{7}\right)$ which correspond respectively to some arbitrarily chosen initial conditions $f_{0}$ and $g_{0}$ belonging to $\Phi_{0}(X \times \Xi)$. Of course, $\tilde{f}$ and $\tilde{g}$ can be obtained as limits, for $n \rightarrow \infty$, of the iterative scheme described in (iii), with $B f_{n}$ replaced by $B\left(f_{0}, f_{n}\right)$ and $B\left(g_{0}, g_{n}\right)$, respectively, and with arbitrary $f_{1}, g_{1} \in \Phi_{\alpha, 1}\left(R_{\tau}^{7}\right) ; \operatorname{see}(3.34)$ for the definition of $B(\cdot, \cdot)$. Now put $f_{1}=f_{0}$ and $g_{1}=g_{0}$. Then by this 'new' iterative scheme and Lemma 3.2 ,

$$
\begin{aligned}
\left\|f_{2}-g_{2}\right\|_{\Phi_{\alpha} ; p} & =\left\|B\left(f_{0}, f_{0}\right)-B\left(g_{0}, g_{0}\right)\right\|_{\Phi_{\alpha} ; p} \\
& \leq\left(1+\varepsilon_{\alpha}(p)\right)\left\|f_{0}-g_{0}\right\|_{\Phi_{\alpha} ; p},
\end{aligned}
$$

and, in a similar manner, we obtain by induction

$$
\left\|f_{n}-g_{n}\right\|_{\Phi_{\alpha} ; p} \leq \sum_{k=0}^{n-1}\left(\varepsilon_{\alpha}(p)\right)^{k}\left\|f_{0}-g_{0}\right\|_{\Phi_{\alpha} ; p} .
$$

In view of (3.33), the required Lipschitz condition follows immediately for $p>\inf \left\{p_{\alpha}\right\}$, where $p_{\alpha}$ is that of the statement (iii), by letting $n \rightarrow \infty$. This ends the proof of the theorem.

REMARKS. The above list of properties of $\tilde{f}$ is not meant to be exhaustive or to include full particulars. For example, we could add a statement on the existence of moments of $\tilde{f}$ and be more specific in assertion ( $i i$ ) of the theorem. Some of the omitted details and properties (in particular, those connected with the concept of the H-function) will be presented in a later paper. 


\section{References}

[1] Leif Arkeryd, "On the Boltzmann equation. Part I: Existence", Arch. Rational Mech. Anal. 45 (1972), 1-16.

[2] Leif Arkeryd, "On the Boltzmann equation. Part II: The full initial value problem", Arch., Rational Mech. Anal. 45 (1972), 17-34.

[3] Leif Arkeryd, "An existence theorem for a modified spaceinhomogeneous, nonlinear Boltzmann equation", Bull. Amer. Math. Soc. 78 (1972), 610-614.

[4] V.I. Arnold and A. Avez, Ergodic problems of classical mechanics (W.A. Benjamin, New York, Amsterdam, 1968).

[5] А.А. Арсеньев [A.A. Arsen'ev], "Задача Ношм для линеаризованного уравнения Больцмана" [The Cauchy problem for the linearized Boltzmann equation], z. Vycisl. Mat. i Mat. Fiz. 5 (1965), 864-882; U.S.S.R. Computational Math. and Math. Phys. 5 (1965), 110-136.

[6] Carlo Cercignani, Mathematical methods in kinetic theory (Plenum Press, New York, 1969).

[7] Sydney Chapman and T.G. Cowling, The mathematical theory of nonuniform gases, third edition (Cambridge University Press, Cambridge, 1970).

[8] J.H. Ferziger and H.G. Kaper, Mathematical theory of transport processes in gases (North-Holland, Amsterdam, London, 1972).

[9] Aleksander Glikson, "On the existence of general solutions of the initial-value problem for the nonlinear Boltzmann equation with a cut-off", Arch. Rational Mech. Anal. 45 (1972), 35-46.

[10] Aleksander Glikson, "On solution of the nonlinear Boltzmann equation with a cut-off in an unbounded domain", Arch. Rational Mech. Anal. 47 (1972), 389-394.

[11] Harold Grad, "Principles of the kinetic theory of gases", Thermodynamik der Gase, 205-294 (Handbuch der Physik, 12. SpringerVerlag, Berlin, Göttingen, Heidelberg, 1958). 
[12] Harold Grad, "Asymptotic theory of the Boltzmann equation", Phys. Fluids 6 (1963), 147-181.

[13] Harold Grad, "Asymptotic theory of the Boltzmann equation, II", Rarefied gas dynamics, Volume I, 26-59 (Proc. Third Internat. Sympos. Rarefied Gas Dymamics, Paris, 1962. Academic Press, New York, London, 1963).

[14] Harold Grad, "Asymptotic equivalence of the Navier-Stokes and nonlinear Boltzmann equations", Applications of nonlinear partial differential equations in mathematical physics, 154-183 (Proc. Sympos. Appl. Maths. Amer. Math. Soc., New York City, 1964, Volume 17. Amer. Math. Soc., Providence, Rhode Island, 1965).

[15] Harold Grad, "Solution of the Boltzmann equation in an unbounded domain", Comm. Pure Appl. Math. 18 (1965), 345-354.

[16] J.-P. Guiraud, "Gas dynamics from the point of view of kinetic theory", Applied Mechanics, Proc. Thirteenth Internat. Congress Theoretical and Appl. Mech., Moscow, 1972, 104-123 (SpringerVerlag, Berlin, Heidelberg, New York, 1973).

[17] J.-P. Guiraud, "An $H$ theorem for a gas of rigid spheres in a bounded domein", Colloques Internationaux C.N.R.S., No. 236, Théories cinétiques classiques et relativistes (1975), 29-58.

[18] E. Kamke, Differentialgleichungen reeller Funktionen (Chelsea, New York, 1947).

[19]* M.N. Kogan, Rarefied gas dynamics (Plenum Press, New York, 1969).

[20] M.A. Krasnosel'skiT, The operator of translation along the trajectories of differential equations (Translations of Mathematical Monographs, 19. Amer. Math. Soc., Providence, Rhode Island, 1968).

[21]* Michal Kunc, "Détermination de la fonction de distribution des vitesses moléculaires dugaz en mouvement stationnaire par la méthode demographique", Arch. Mech. Stos. 9 (1957), 731-737.

[22] Dietrich Morgenstern, "Analytica] studies related to the MaxwellBoltzmann equation", J. Rational Mech. Anal. 4 (1955), 533-555. 
[23] А.Я. Повзнер [A.Ja. Povzner], "Об уравненин Больцмана в нинетечесной теории газов" [On the Boltzmann equation of the kinetic theory of gases], Mat. Sb. N.S. 58 (1962), 65-86.

[24] G. Scharf, "Functional-analytic discussion of the linearized Boltzmann equation", Helv. Phys. Acta 40 (1967), 929-945.

[25] G. Scharf, "Normal solutions of the linearized Boltzmann equation", Helv. Phys. Acta 42 (1969), 5-22.

[26] Yasushi Shizuta, "On the classical solutions of the Boltzmann equation", Comm. Pure Appl. Math. (to appear).

[27] Yasushi Shizuta, "The existence and approach to equilibrium of classical solutions of the Boltzmann equation", Comm. Math. Phys. (to appear).

[28] C. Truesdell and R.G. Muncaster, Book on Maxwell's theory, in preparation.

[29] Seiji Ukai, "On the existence of global solutions of mixed problem for non-linear Boltzmann equation", Proc. Japan Acad. 50 (1974), $179-184$.

[30] С.В. Валландер [S.V. Vallander], "Новые нинетичесние уравнения в теорин одноатомных газов" [New kinetic equations in the theory of monatomic gases], DokZ. Akad. Nauk SSSR 131 (1960), 58-60; Soviet Physics Dokl. 5 (1960/1961), 269-271.

[31] E. Wild, "On Boltzmann's equation in the kinetic theory of gases", Proc. Combridge Philos. Soc. 47 (1951), 602-609.

[32]* D.R. Willis, "A study of some nearly-free molecular flow problems" (PhD thesis, Princeton University, 1958); or Aero. Eng. Report No. 440, Princeton University, 1958.

[33] D. Roger Willis, "On the flow of gases under nearly free molecular conditions", AFOSR TN 58-1093, Report No. 442, AD 207594 (1958).

* These references are at present unavailable to the author, and may, therefore, not be entirely accurate. Editor.

Department of Mathematics, University of New England, Armi dale, New South Wales. 